\title{
Blocking in landmark-based search in honeybees
}

\author{
KEN CHENG \\ Macquarie University, Sydney, New South Wales, Australia \\ and \\ MARCIA L. SPETCH \\ University of Alberta, Edmonton, Alberta, Canada
}

\begin{abstract}
Two experiments tested blocking in landmark-based search in honeybees. Honeybees in the experimental group were trained in Phase 1 with a single landmark in a constant spatial relation to the target (sugar water). In the compound training second phase, the landmark used in Phase 1 (blocking landmark) and a new landmark (blocked landmark) were presented at constant spatial relations to the target. The blocking and blocked landmarks differed from each other in color and position, and the blocking landmark retained the same spatial relationship to the target as in Phase 1. In Experiment 1, the control group experienced only Phase 2 training with two landmarks. In Experiment 2, the control group was trained with a different landmark in a different position in Phase 1. Blocking was found in both cases.
\end{abstract}

Blocking is by now a classic phenomenon in classical conditioning (Kamin, 1969). In blocking, what would have been learned about one conditioned stimulus (CS) is not learned or not expressed because of experience with another CS. In the first phase of training, a CS alone, CSA, is paired with the unconditioned stimulus (UCS). In the second phase of training, two CSs, CSA and CSB, are together paired with the UCS (fear-provoking shock). Kamin's results showed that rats feared CSA, but not CSB. Another group trained solely with Phase 2, CSA and CSB together, feared CSB.

As part of a program of research testing general laws of learning in the spatial domain, we report experiments here in which we tested for blocking effects in landmarkbased search in honeybees. Getting back to a place is an important problem to solve for many animals. Mechanisms dedicated to solving the problem are well documented (for reviews, see Gallistel, 1990; Healy, 1998; Wehner, Lehrer, \& Harvey, 1996). Some of these mechanisms appear to be highly specialized for the spatial domain. For example, the honeybee forager uses a sequence of place-finding servomechanisms for getting from her hive to a foraging spot (for reviews, see Cheng, 2000a; Collett \& Zeil, 1998). The sequence starts with a vector getting the bee from the hive to the vicinity of the target (Dyer, 1991; Wehner, Bleuler, Nievergelt, \& Shah, 1990; Wehner \& Menzel, 1990). Next, the bee beacons toward

The research reported here was supported by a grant from the Australian Research Council to K.C. Thanks are due Dagong Zhang for data collection, Dagong Zhang and Jamie Berry for data analysis, and Mark Peterson for hive maintenance. Correspondence about this article should be addressed to K. Cheng, Department of Psychology, Macquarie University, Sydney, NSW 2109, Australia (e-mail: kcheng@axon.bhs. mq.edu.au). recognized landmarks near the target. Somewhere during the beaconing trajectory, she may veer off on a sensorimotor vector toward the target location (Cheng, 1999b; Collett \& Rees, 1997). This trajectory is sensorimotor in that a particular (sensory) view of the landmark is associated with a particular (motor) trajectory to head to the target. Finally, on failing to see the target, the bee may engage in image matching, a process in which she attempts to place herself at the location at which the surrounding landmarks appear as they did at the remembered target location (Cartwright \& Collett, 1982, 1983; Cheng, 1999b; Collett \& Rees, 1997). The positions of landmarks are defined retinally, in terms of where they should appear on the retina. In order to dispense with having to translate retinal coordinates into some other coordinate system, the honeybee flies facing stereotypical directions while searching near the target (Cheng, 1999b; Collett \& Baron, 1994; Frier, Edwards, Smith, Neal, \& Collett, 1996). Given the occurrence of specialized processes that appear to be dedicated to the problem of place finding, an interesting question is whether general processes-specifically those that are considered general laws of learning within nonspatial domains - also operate for place finding.

Recent studies with mammals and birds have provided evidence for a number of general laws of learning in the spatial domain. For example, we have found spatial generalization gradients in both pigeons (Cheng, Spetch, \& Johnston, 1997) and humans (Cheng \& Spetch, 2000). In these experiments, a reward-giving target location was presented to the subjects during training, and then unrewarded tests at a range of locations along one dimension of space were occasionally mixed with training trials. The form of the resulting generalization functions was consistent with Shepard's law of generalization (Shepard, $1958,1987)$, previously supported only in nonspatial domains. Recently, Cheng (1999c, 2000b) reported similar 
generalization gradients in honeybees, indicating remarkable cross-species generality in these functions.

In experiments with pigeons and humans, we have also tested the peak shift phenomenon (Cheng \& Spetch, 2000; Cheng et al., 1997) by training with both a target location $(\mathrm{S}+)$ and a nonrewarding location $(\mathrm{S}-)$ that was near $\mathrm{S}+$ during training. The generalization gradient obtained from both pigeons and humans showed higher responding on the side of $\mathrm{S}+$ away from $\mathrm{S}-$, an effect called area shift (Rilling, 1977). The results parallel the pattern found for similar $\mathrm{S}+/ \mathrm{S}-$ training in other dimensions of experience (e.g., wavelength of light, Hanson, 1959).

Another general learning phenomenon is overshadowing. In overshadowing, a CS that supports good conditioning when trained alone with the UCS shows less conditioning when presented in compound with another CS (see, e.g., Kaye, Gambini, \& Mackintosh, 1988; Mackintosh, 1976). In the spatial domain, overshadowing has been found for rats (March, Chamizo, \& Mackintosh, 1992; Sánchez-Moreno, Rodrigo, Chamizo, \& Mackintosh, 1999), as well as for pigeons and humans (Spetch, 1995). March et al. (1992) tested rats on the radial maze task and found that intramaze cues and extramaze cues overshadowed each other reciprocally. SánchezMoreno et al. (1999) tested rats in the Morris water maze and found reciprocal overshadowing between an auditory cue and visual landmarks. Spetch (1995) tested pigeons and humans, using computer-generated landmarks presented on a monitor. The target was found at the same place for both species with respect to a set of landmarks. Accuracy of performance using overshadowed and nonovershadowed landmarks was compared. The two kinds of landmarks stood at the same absolute distance from the target, but the overshadowed landmark was presented together with another landmark that was closer to the target. Both species performed better when tested with the nonovershadowed landmark alone than with the overshadowed landmark alone.

The classic blocking effect has also been found in the spatial domain in several studies with rats (Biegler \& Morris, 1999; Chamizo, Sterio, \& Mackintosh, 1985; Roberts \& Pearce, 1999; Rodrigo, Chamizo, McLaren, \& Mackintosh, 1997). Rodrigo et al. (1997) found spatial blocking in rats in a swimming task. The rats had to find a submerged platform in a circular pool by using landmarks that the experimenters provided. Other landmarks were blocked from view by a curtain surrounding the pool. If the rats were first trained to locate the platform by using three landmarks, they then failed to use a fourth additional landmark. Biegler and Morris (1999) also found spatial blocking among an array of discrete landmarks in an open field arena. After training to find food using a set of landmarks, the rats noticed and explored an added landmark, but they failed to use it in searching for the hidden food.

To the best of our knowledge, neither overshadowing nor blocking effects have been tested in landmark-based search tasks with invertebrates. However, both effects have been reported in honeybees within other paradigms. Overshadowing in honeybees has been extensively investigated by Couvillon, Bitterman, and colleagues (e.g., Couvillon \& Bitterman, 1989; Couvillon, Klosterhalfen, \& Bitterman, 1983; Couvillon, Mateo, \& Bitterman, 1996; for a review, see Bitterman, 1996). Blocking effects in honeybees have been investigated within two paradigms: conditioned proboscis extension and foraging for sugar water. In the conditioning of proboscis extension, the honeybee is strapped in a harness to keep it in place. The UCS consists of tactile presentations of sugar water to the antennae. The bee responds by extending its proboscis to drink the sugar water. Both scent and another mechanical stimulus have been used as CSs (for a review, see Hammer \& Menzel, 1995). After two conditioning trials, the animals generally extend the proboscis on encountering the CS alone. Smith and Cobey (1994) found blocking when they used odors as blocking and blocked CSs.

Funayama, Couvillon, and Bitterman (1995) tested blocking in honeybees in choice tests. In six experiments, honeybees were presented with choice tests in extinction between a blocked stimulus and a control stimulus. The blocked stimulus had been trained in compound with another cross-modal stimulus that had signaled reward (rather than nonreward) or more reward (rather than less reward). The control stimulus had been trained in compound with another cross-modal stimulus that had signaled no reward or less reward. Symbolically, one experiment (Experiment 1) could be represented as A+/B followed by $\mathrm{AX}+$ and $\mathrm{BY}+$, followed by choice tests in extinction with $\mathrm{X}$ and $\mathrm{Y}$. In all these experiments, Funayama et al. looked for the blocking of odors by colors, whereas in one they also examined blocking of colors by odors. Blocking was not found: The honeybees sought out the blocked stimuli as much as they did the control stimuli, even when the possibility of within-compound associations was controlled for. The results were consistent with the independent learning of odors and colors.

Couvillon, Arakaki, and Bitterman (1997) studied blocking in honeybees with combinations of color, odor, and position. Position was defined by a landmark adjacent to the target the bees were to aim for. In each of four experiments, a control group was compared with a blocking group. The authors found blocking for color-color compounds, odor-odor compounds, and color-landmark compounds, but not for odor-landmark compounds.

In more recent experiments, Couvillon and Bitterman (1999) have found that the occurrence of blocking depends on the salience of the stimuli used, and that it often shows asymmetric patterns. That is, sometimes, A blocks B but B does not block A. Under some circumstances, intermodal blocking can be found.

Phenomena such as overshadowing and blocking have been called phenomena of cue competition. In our brief review here, we have described various animals being tested in various paradigms. To set out the significance of the experiments reported here, it is important to make 
some conceptual distinctions in the ways in which cues may be used to guide an animal's behavior. In the more traditional classical conditioning in the temporal domain, the cues help the animal to decide when to search, whereas in the spatial domain, the cues help the animal decide where to search.

Within the spatial domain, a further distinction has been emphasized - namely, spatial learning that involves approach or avoidance of a particular place based on a beacon, and spatial learning that involves using spatial cues to locate and navigate toward a different location. According to O' Keefe and Nadel (1978), the former is based on a guidance system that is associative in nature: Animals learn to approach or avoid a particular cue. Learning to find a specific, undifferentiated place on the basis of spatial cues that are not right at the goal requires more than this, and according to O' Keefe and Nadel it involves forming and updating a cognitive map of the environment. This kind of learning, called locale or place learning, is thought to be a fundamentally different kind of learning than guidance learning. Strong support for the distinction has been provided by studies showing that hippocampal lesions impair rats' ability to learn the new location of an invisible platform (locale learning) but not their ability to find a visible platform (guidance, or beacon learning; see, e.g., Morris, Garrud, Rawlins, \& O'Keefe, 1982; for a review, see Leonard \& McNaughton, 1990). An analogous effect is found in food-storing birds with hippocampal lesions (e.g., Sherry \& Vaccarino, 1989), who have trouble remembering the location of stored caches of food, but can still learn to approach a particular cue. Given this distinction, guidance learning is reasonably expected to follow principles of Pavlovian conditioning, but locale learning might not (Morris, 1981). In this theoretical context, the previously mentioned demonstrations of cue competition effects in place learning by vertebrates have been of considerable theoretical importance.

Cues may play different roles in honeybee foraging as well. The conditioning of proboscis extension in honeybees is a good example of temporal conditioning (see, e.g., Smith \& Cobey, 1994). The "where problem" in this case is small; the food is after all right in front of the bee's head, both in the conditioning experiment and in her real life. The major problem for foraging efficiency is that of sticking out the proboscis at the appropriate time. Odor CSs, again both in the experiment and in real life, help the forager make that "when decision."

In the foraging experiments of Couvillon, Bitterman, and colleagues (e.g., Couvillon et al., 1997), on the other hand, the food was there at all times for the bees. The forager's problem was that of deciding where to go to find the food. Because the honeybee takes several steps in finding a foraging spot (Cheng, 2000a), cues in the spatial domain can help in different stages of the search. Accordingly, it is important to consider the kind of spatial cue, and the type of spatial process activated. In the Couvillon et al. experiments, the competing cues were right at the target location. For example, in Experiment 3 of Couvillonet al. (1997), the food was on a petri dish, while a color strip on the dish and a block immediately adjacent to the dish were the competing cues. Hence, cue control over beaconing behavior was examined.

In our experiments, the target was at a small distance from one or more landmarks. The food was again there all the time, and when to search was not the problem. In the search for food, beaconing was not enough: The food was not right at the landmark. Learning simply to approach the landmark would not suffice, because it would not get the bee to the correct direction from the landmark. The forager had also to use the last two steps: sensorimotor vector and image matching. Hence, in these experiments we tested cue competition over these aspects of spatial search behavior. To the best of our knowledge, this issue has not been examined in honeybees to date. Our paradigm is most analogous to the spatial cue competition paradigms tested on vertebrates, in which cues are at some distance from the target. Whether on a touch screen (e.g., Spetch, 1995), in a swimming pool (e.g., Rodrigo et al., 1997), or on an open field (e.g., Biegler \& Morris, 1999), the problem is to search at the right place with respect to one or more landmarks.

Thus, our investigations of blocking in landmark-based search in honeybees are theoretically interesting from three perspectives. First, these studies extend recent work by ourselves and others that is concerned with testing general laws of learning in the spatial domain. Determining the extent to which laws of learning apply in the spatial domain has implications for theories of spatial cognition (see, e.g., O' Keefe \& Nadel, 1978). Second, our investigations are interesting from a comparative perspective. Given that some of the specialized place finding mechanisms (e.g., image matching) appear to differ between vertebrates and invertebrates, it is of interest to determine whether blocking in the spatial domain occurs in bees as it does in rats. Third, our work extends the recent investigations of blocking in bees.

\section{EXPERIMENT 1}

Experiment 1 provided an initial test of blocking in landmark-based search. In the first phase, bees in the blocking group were trained with a single landmark, the blocking landmark. A blue landmark was chosen as the blocking landmark because it was the less preferred color in past experiments (Cheng, 1999a). In Phase 2, the target was in between a blue and a yellow (the blocked) landmark. The target-blocking-landmark relation was the same in both phases (Figure 1). On a crucial test, the blocked landmark was presented alone. On another test, the two landmarks were put in conflict; we recorded whether the bees followed the blocking landmark or the blocked landmark more. The control group was given only Phase 2 training prior to the tests. Thus, if training with the blue landmark alone blocked control by the yellow landmark, then control by the yellow landmark during 


\section{Design for Experiment 1}

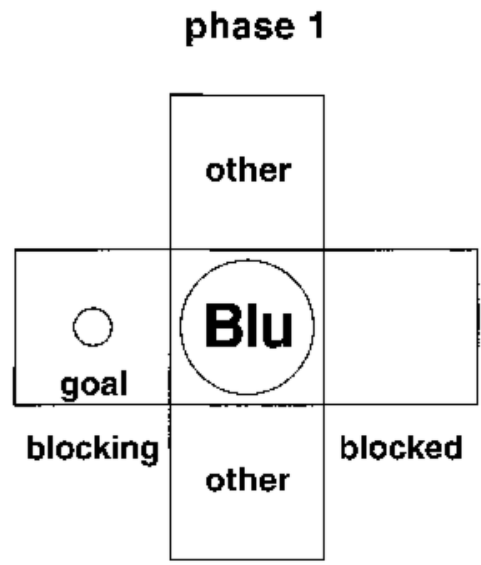

$10 \mathrm{~cm}$

phase 2

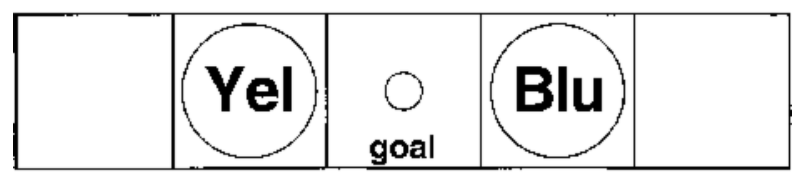

blocking

middle

blocked

Figure 1. Landmark setup used in Experiment 1. The landmarks (large circles) were a light yellow cylinder (Yel) and a light blue cylinder (Blu), both $9 \mathrm{~cm}$ in diameter and height. The goal was a cap filled with sugar water. The entire training array was moved from trial to trial, so that the landmarks were the only valid spatial predictor of the exact location of reward. For some bees, the goal was to the right of the blocking landmark in Phase 1. Bees approached the table with the setup from the bottom of the figure.

the tests should be greater for the control group than for the blocking group.

\section{Method}

\section{Animals and Materials}

Honeybees (Apis mellifera) for the experiments lived in a maintained hive near the lab. A feeding station providing sugar water was set up for the bees on a table outside the entrance window of the lab. Experimental animals (10 bees in each group, run in two lots of 5) were recruited from the feeding plate. The plate was placed near the entrance to the lab, and reward-strength sugar water (two parts sugar to three parts water) in a translucent bottle cap $(2.5 \mathrm{~cm}$ in diameter) was placed at the entrance window. The bees that found this stronger sugar water typically returned to the lab entrance. Returning bees were individually marked for identif ication.

\section{Setup}

Experimental bees entered the lab through a 2-cm square hole in a north-facing window. On the bottom of the hole sat a Perspex gangplank painted with stripes of red nail polish. A Perspex "gate" allowed entrance control for experimenters. Insects walked through the hole on the gangplank and then had to fly to a table for their final destination and reward in the lab. The long edge of the table $(120 \times 75 \times 73 \mathrm{~cm}$ high $)$ parallelled the wall under the window, and the table was covered with white poster paper with yellow squiggles drawn on it with a highlighting pen. The squiggles helped to stabilize the flights of honeybees over the otherwise featureless surface; they were similarly used in past studies (Cartwright \& Collett, 1982, 1983; Cheng, 1998a, 1998b, 1999a, 1999b; Cheng, Collett, Pickhard, \& Wehner, 1987; Cheng, Collett, \& Wehner, 1986). Also drawn on the table, in pencil, was a grid of 5.2-cm squares. These were used for putting the target in position and for scoring data. After the forager drank her fill of the reward, she flew to the window, and was released through a swinging door (10-cm square) above the entrance.

The lab room contained plenty of cues indicating direction. These included a stand for the black and white video camera above the table, which straddled the two short sides of the table, an experimenter at a seat near the table, a window, three doors, video equipment, and other furniture. Overhead fluorescent lights (full spectrum tubes in translucent covers) lit the room.

\section{Training arrays}

Landmarks were cylindrical bottles ( $9 \mathrm{~cm}$ diameter, $9 \mathrm{~cm}$ high). One was wrapped in light yellow poster paper and the other was wrapped in light blue poster paper (Figure 1). The same colors were used in various previous experiments (Cheng, 1998a, 1998b, 1999a, $1999 \mathrm{~b}, 1999 \mathrm{c})$. The bees in the blocking group were trained with two different arrays in two different phases of the experiment (Figure 1). The bees in the control group received only the second phase of training.

\section{Procedure}

Experimentation took place between 0900 and 1630. Five bees in the same condition were trained at the same time in one lot. Then a different lot of 5 in a different condition were trained and tested, and so on. On early trials, the reward was at the gangplank at the entrance, and a bee was moved from the entrance window to the correct place on the table, with the initial training setup in place (Phase 1 setup for the blocking group and Phase 2 setup for the control group). After a few such trials, the forager was released at a small distance from the goal. Then she was released beyond the edge of the table. After this initial training, the bees had to fly directly from the gangplank to the table.

Blocking group. Training and testing took place in two phases. During Phase 1, the bees received training with the blue landmark alone. The landmark was on the table, in the middle of an area of four square grid units. For half of the bees, the target (bottle cap of sugar water) was on the left (east) side of the landmark along the length of the table; for the remaining 5 bees, it was on the right (west), with left and right defined from the entrance to the lab. The center of the target was at an intersection of grid lines, $5.9 \mathrm{~cm}$ to the nearest edge of the landmark. In the second phase, both the blue and the yellow landmarks were on the table, on both sides of the target along the length of the table, also with the target at an intersection of grid lines. Target-landmark distance was the same as in Phase 1. For all bees, the blue landmark was on the same side of the target in Phases 1 and 2. Throughout experimentation, the location of the training array (landmarks and target) on the table changed from trial to trial. Thus, the only valid predictors of the exact location of the target were the landmarks. The bees were trained for 20 trials in each phase and then were tested. Two of each of four kinds of tests were given after training to each bee in each phase. The crucial tests were single-landmark tests with the yellow (blocked) landmark only. Two kinds of two-landmark tests were also given, one in which the landmark arrangement matched that found on the Phase 2 training trials (control tests), and one in which the two landmarks were presented in reversed positions to those used in Phase 2 train- 
ing (reversed tests). For the latter tests, this meant that the correct locations with respect to the blue and yellow landmarks were outside of the array, at two different locations. In addition, tests with only the blue (blocking) landmarks were also given. Although bees might be trained simultaneously, they were always tested individually. Others were shut out by the gate or else temporarily captured in a matchbox. On a test, the cap of sugar water was absent. The location of the landmark also varied, but it was centered under the video camera. The bee's behavior was videotaped for $60 \mathrm{sec}$, from the time she entered the test area. She was then given a regular training trial. Two more training trials intervened before another test trial.

Control group. The procedure used for this group was identical to that of the blocking group, except that Phase 1 of training and testing was omitted. Thus, the control group did not receive the singlelandmark training phase and received two-landmark training only prior to the tests.

\section{Data Analysis}

The videotape record of each test was fed into a computer. The amount of time (frames divided by frame rate) that the bee spent in critical regions was counted. The critical regions are shown in Figure 1 , each square comprising four square grid units. On onelandmark tests, which included the crucial test with the blocked landmark, we scored four equal-sized locations on four sides of the landmark. The training location in the one-landmark training phase (Phase 1) was called the blocking location, and the location on the opposite side of the landmark was called the blocked location. The other two locations were at $90^{\circ}$ between the blocking and blocked locations. The bees had no theoretical reason to search at these other locations, and counting the amount of time spent there gave a baseline measure. We averaged the two locations, and called it "other." Thus, the location factor was divided into three levels: blocking, blocked, other.

On two-landmark tests, the outside square on the side of the blocking (blue) landmark was called the blocking location. The outside square on the opposite side (beside the yellow, blocked landmark) was called the blocked location. Note that on reversed tests, these outside locations matched the spatial relation found in Phase 2 training between the target and the nearest landmark. The location in the middle was called the middle. Statistical tests throughout were considered signif icant at $p<.05$.

Analysis was done by a semi-automatic movement analysis system developed in house. The program was developed from the Image software of the National Institute of Health. Image is available at http://rsb.info.nih.gov/ nih-image/Default.html. This movement analysis system has been used in recent studies (Cheng, 1998a, 1999b). The program subtracts from each video image the average of all frames. This leaves the bee as the remaining object. Checking a few finished tests revealed no errors in the form of non-bee objects being identified. Marking reference locations and exporting all data to a spreadsheet allowed us to determine whether the bee was in any of the regions of interest on each frame.

\section{Results and Discussion}

\section{Blocked Landmark}

We begin with the classic test used for blocking, the test with the blocked (yellow) landmark. The critical comparison is that between the blocking group and the control group for the tests that followed Phase 2 (Table 1). Table 1 shows that although both groups searched most at the correct location, the control group showed more concentrated search at the correct location than did the blocking group. A mixed-model analysis of variance (ANOVA) revealed significant main effects of group
$[F(1,16)=8.05]$ and location $[F(2,32)=21.13]$, and importantly, a significant interaction between group and location $[F(2,32)=5.02]$. To check whether bees in the blocking group showed any evidence of using the yellow landmark, we compared the amount of searching at the appropriate location across the two phases on tests with the blocked landmark. The bees searched almost five times as much in Phase 2 as in Phase $1[F(1,9)=17.6]$, a highly significant difference that was consistent in every individual. Thus, the blocking group did search at the appropriate location for the blocked landmark, although they did not do so as much as the control group.

\section{Other Tests}

On the reversed tests, in which the dictates of the two landmarks were put in conflict, honeybees in the two groups behaved differently (Table 1). Honeybees in the blocking group preferred to follow the dictates of the blocking landmark during the reversed tests, whereas the control group searched about equally at the three locations. A mixed-model ANOVA of Phase 2 data revealed significant effects of location $[F(2,32)=5.23]$ and location $\times$ group interaction $[F(2,32)=4.78]$. The blocking group behaved similarly in Phase 1 and in Phase 2 (results not shown).

On two-landmark tests in which the landmark configuration was the same as during the second phase of training (control tests), bees in both groups searched most at the appropriate middle location (Table 1). Bees in the control group, however, showed more concentrated search than did bees in the blocking group. A mixed-model ANOVA revealed a significant main effect of location $[F(2,32)=57.51]$ and a significant location $\times$ group interaction $[F(2,32)=4.23]$.

On tests with the blue (blocking) landmark only, honeybees in both groups searched appropriately in the correct location according to the blue landmark (Table 1). A mixed-model ANOVA revealed a significant main effect of location $[F(2,32)=25.27]$, but the main effect of group and the interaction between group and location were not significant. It also revealed a significant location $X$ version (whether the blue landmark was to the east or to the west of the target) interaction $[F(2,32)=4.66]$. Inspection of the data revealed the same pattern of results for both versions, with bees in one version searching more in the target area than bees in the other version.

In Experiment 1, we demonstrated blocking in landmarkbased learning in bees, using one of the standard control groups for assessing blocking, an AB control group that had no prior experience with the blocking landmark. The blocking group used the blocked landmark less well than did the control group, but both groups used the blocking landmark equally well.

\section{EXPERIMENT 2}

Bees that were first trained to find food using a single blue landmark showed less control by an added yellow 
Table 1

Mean Duration of Searching (in Seconds) in

Designated Areas on 60-Sec Tests in Phase 2 of Experiment 1 and Mean Proportion of Searching in Target Area in Phase 2 of Experiment 2

\begin{tabular}{|c|c|c|c|c|c|c|c|}
\hline Test & Group & $M$ & SEM & $M$ & $S E M$ & $M$ & SEM \\
\hline Experiment 1 & & \multicolumn{2}{|c|}{$\underline{\text { Blocking Side }}$} & \multicolumn{2}{|c|}{ Blocked Side } & \multicolumn{2}{|c|}{ Other } \\
\hline \multirow[t]{3}{*}{ Blocked LM } & Blocking & 2.7 & 0.55 & 4.0 & 0.66 & 2.4 & 0.28 \\
\hline & Control & 2.7 & 0.37 & 6.8 & 0.72 & 3.8 & 0.45 \\
\hline & & \multicolumn{2}{|c|}{ Blocking Side } & \multicolumn{2}{|c|}{ Middle } & \multicolumn{2}{|c|}{ Blocked Side } \\
\hline \multirow[t]{3}{*}{ Reversed } & Blocking & 5.4 & 0.75 & 3.0 & 0.41 & 1.7 & 0.37 \\
\hline & Control & 4.2 & 0.82 & 5.3 & 0.59 & 3.8 & 0.75 \\
\hline & & \multicolumn{2}{|c|}{ Blocking Side } & \multicolumn{2}{|c|}{ Middle } & \multicolumn{2}{|c|}{ Blocked Side } \\
\hline \multirow[t]{3}{*}{ Control } & Blocking & 2.1 & 0.38 & 7.7 & 0.77 & 1.5 & 0.30 \\
\hline & Control & 2.2 & 0.35 & 12.0 & 1.70 & 1.3 & 0.24 \\
\hline & & \multicolumn{2}{|c|}{ Blocking Side } & \multicolumn{2}{|c|}{ Blocked Side } & \multicolumn{2}{|c|}{ Other } \\
\hline \multirow[t]{2}{*}{ Blocking LM } & Blocking & 7.2 & 0.95 & 3.2 & 0.27 & 3.9 & 0.32 \\
\hline & Control & 6.1 & 0.67 & 3.3 & 0.48 & 3.7 & 0.28 \\
\hline \multirow[t]{2}{*}{ Experiment 2} & & \multicolumn{4}{|c|}{$\begin{array}{c}\text { Proportion of Search } \\
\text { in Target Area } \\
\end{array}$} & & \\
\hline & & & $M$ & $S E N$ & & & \\
\hline \multirow[t]{2}{*}{ Blocked LM } & Blocking & & 0.25 & 0.02 & & & \\
\hline & Control & & 0.36 & 0.02 & & & \\
\hline
\end{tabular}

landmark than did bees that were trained only with both landmarks. This constitutes a classic demonstration of a blocking effect. Experiment 2 was designed to replicate this result and to include a different control commonly used in experiments on blocking to ensure that the blocking effect is due specifically to the presence of a trained landmark in Phase 2. A blocking group replicated the blocking group of Experiment 1, except that only tests with the blocked landmark were given. The control group was also trained and tested in two phases, the second of which was identical to that of the blocking group. In the first phase, however, they received training with a landmark that is different in color from both landmarks encountered in Phase 2. The position of the landmark in Phase 1 also differed from those found in Phase 2. As a result, the Phase 1 landmark did not block in either the positional or the color dimension. The condition constitutes the classic $\mathrm{C} / \mathrm{AB}$ control.

\section{Method}

The subjects came from the same hive as in Experiment 1, and they were tested in the same lab. The gangplank at the entrance to the lab was now uniformly red in color, being covered by red poster paper. In addition to the yellow and blue landmark used previously, an additional landmark of the same size, wrapped in red poster paper, was used in some conditions. The yellow landmark once again served as the blocked landmark. The landmark (blue or red) used in Phase 1 training varied across bees in each group. For each group, a subset of the many possible permutations of landmark color and target position were selected as the Phase 1 and Phase 2 training arrangements. These are illustrated in Figure 2, along with the number of subjects completing each condition.

All groups were trained and tested in two phases, with 20 training trials in each phase. After training in each phase, three tests with the yellow landmark were given to each subject. Otherwise, the procedure followed that of Experiment 1. For the dependent variable, the number of frames in which the bee appeared in the target area defined by the yellow landmark was counted. This was divided by the number of frames in which the bee appeared in equivalent areas in all four cardinal directions around the landmark. This measure yields a proportion of searching in the target area, with .25 as chance level or equal searching in all directions around the landmark. We avoided using different locations around the test landmark as a factor, because with the different versions of setups used in this experiment, classifying locations other than the target location was problematic.

\section{Results and Discussion}

The crucial results from Phase 2 are displayed in Table 1. We excluded one control animal who searched very little in the target area in Phase 2 (more than three standard deviations below the mean of the other 8 control animals). The control groups searched proportionally more in the target area of the blocked landmark than did the blocking groups $[F(1,12)=6.24]$, replicating the results of Experiment 1. Hence, blocking in landmark-based search was found in honeybees using both a -/AB control group and a $\mathrm{C} / \mathrm{AB}$ control group.

To check whether the blocking group used the yellow landmark at all in searching, we compared their performance across the two phases. The bees searched three times more at the appropriate location in Phase 2 as in Phase 1 -highly significant results $[F(1,7)=102]$ that were consistent in every individual. Comparing the proportion of search time in the target area yielded a similar pattern of more searching in the target area in Phase 2 $[F(1,7)=9.93]$. It should be noted that both blocking and control bees searched in the target area at lower than chance levels in Phase 1, presumably because they had never been trained with the yellow landmark and they tended to search to the side of the landmark that had been 


\section{Blocking: $A / A B$}

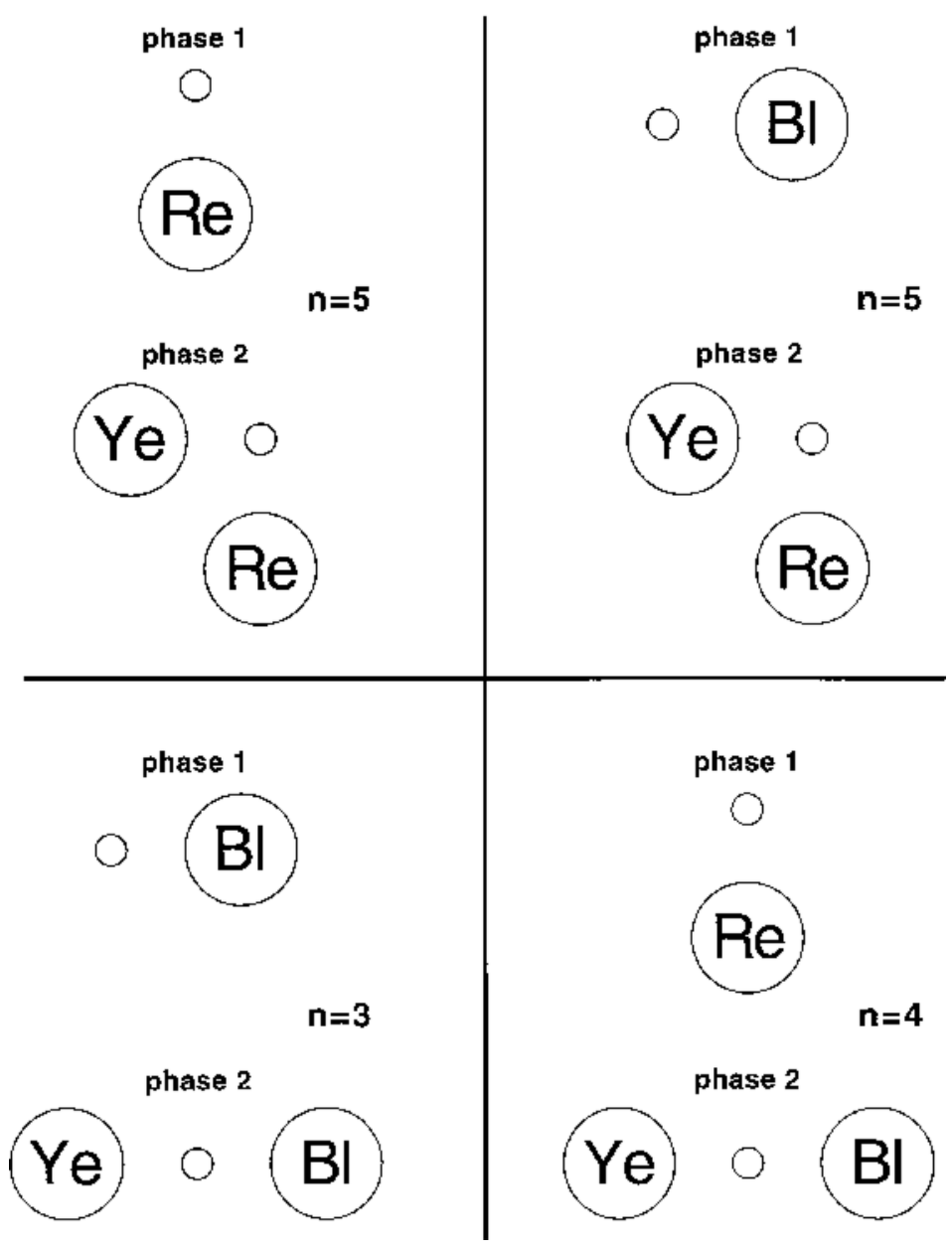

Figure 2. Landmark setups used in Experiment 2. The landmarks (large circles) were a light yellow cylinder (Ye), a light blue cylinder (BI), and a red cylinder $(\mathrm{Re})$, each $9 \mathrm{~cm}$ in diameter and height. The goal was a cap filled with sugar water. The entire training array was moved from trial to trial, so that the landmarks were the only valid spatial predictor of the exact location of reward. Bees approached the table with the setup from the bottom of the figure.

correct for the trained landmark. Therefore, even though the .255 proportion of searching for the blocking group in Phase 2 was close to the chance level, it significantly increased from Phase 1. Hence, although they did not use the blocked landmark as well as the control group did, the blocking group did search more at the appropriate location for the blocked landmark after Phase 2 training.

\section{GENERAL DISCUSSION}

In these experiments on blocking in the spatial domain, honeybees were tested in experiments on tasks of landmark- based searching. The blocking groups were trained with a single landmark (the blocking landmark) in the first phase. In the second phase, they were trained with two landmarks (the blocking and blocked landmarks). The spatial relation of the target to the blocking landmark remained constant across the phases. In Experiment 1, the control group only had Phase 2 training. Experiment 2, on the other hand, replicated the blocking group of Experiment 1 , but a C/AB group was used as a control. This group experienced an irrelevant landmark in an irrelevant position in Phase 1. Blocking was found in both experiments: After Phase 2 training, the blocking group searched 
less in the target area on tests with only the blocked landmark than did control animals.

Our demonstration of blocking in landmark-based search in honeybees extends the range of parallel phenomena found in both searching in space and searching in time. Other parallels between interval timing and landmark-based spatial search have already been noted (Cheng, 1992). It is important to determine how much the spatial and temporal domains share in principles of learning and memory. To the extent that these principles are similar, it would suggest a common underlying neurophysiological mechanism for coding spatial and temporal information. Indeed, we have suggested this common coding on the basis of results indicating that both pigeons and humans average the dictates of elapsed time and spatial position (Cheng, Spetch, \& Miceli, 1996). Still to be determined in pursuing such spatial/temporal parallels are the conditions that engender blocking and cue competition. Miller, Matute, and colleagues have recently found that biologically significant stimuli are less subject to blocking and other phenomena of cue competition than biologically insignificant stimuli (Denniston, Miller, \& Matute, 1996; Miller \& Matute, 1996; Oberling, Bristol, Matute, \& Miller, 2000). Whether this also holds in the spatial domain remains an open question. The demonstrations of cue competition in the spatial domain have all used innocuous landmark stimuli, such as graphics on a computer monitor or plastic cylinders in an arena. Such landmarks would seem to have little inherent biological significance. Likewise, the landmarks that we used, colored cylinders, would seem to have little inherent biological significance for honeybees. Are salient landmarks, such as very large ones, more immune to cue competition? Is an animal's home, of inherent biological significance, also more immune to cue competition?

In sum, our results add to a body of general laws of learning in the spatial domain. These include: (1) overshadowing, which has been found in rats (March et al., 1992; Sánchez-Moreno et al., 1999) as well as humans and pigeons (Spetch, 1995); (2) blocking, which has been found in rats (Biegler \& Morris, 1999; Chamizo et al., 1985; Roberts \& Pearce, 1999; Rodrigo et al., 1997); spatial generalization, which has been found in pigeons (Cheng et al., 1997), humans (Cheng \& Spetch, unpublished results), and bees (Cheng, 1999c, 2000b); and (4) peak shift, which has been found in pigeons (Cheng et al., 1997) and humans (Cheng \& Spetch, 2000). Special dedicated mechanisms do operate in the spatial domain. Honeybees, for example, use a sequence of servomechanisms for finding a place (Cheng, 2000a). But increasingly, it looks as if general laws of learning also apply in the spatial domain.

\section{REFERENCES}

Biegler, R, \& Morris, R. G. M. (1999). Blocking in the spatial domain with arrays of discrete landmarks. Journal of Experimental Psychology: Animal Behavior Processes, 25, 334-351.
Bitterman, M. E. (1996). Comparative analysis of learning in honeybees. Animal Learning \& Behavior, 24, 123-141.

Cartwright, B. A., \& Collett, T. S. (1982). How honeybees use landmarks to guide their return to a food source. Nature, 295, 560-564.

Cartwright, B. A., \& Collett, T. S. (1983). Landmark learning in bees. Journal of Comparative Physiology A, 151, 521-543.

Chamizo, V. D., Sterio, D., \& Mackintosh, N. J. (1985). Blocking and overshadowing between intra-maze and extra-maze cues: A test of the independence of locale and guidance learning. Quarterly Journal of Experimental Psychology, 37B, 235-253.

Cheng, K. (1992). Three psychophysical principles in the processing of spatial and temporal information. In W. K. Honig \& J. G. Fetterman (Eds.), Cognitive aspects of stimulus control (pp. 69-88). Hillsdale, NJ: Erlbaum.

Cheng, K. (1998a). Distances and directions are computed separately by honeybees in landmark-based search. Animal Learning \& Behavior, 26, 455-468.

Cheng, K. (1998b). Honeybees (Apis mellifera) remember two neartarget landmark constellations. Learning \& Motivation, 29, 435-443.

Cheng, K. (1999a). Landmark-based spatial search in honeybees: I. Use of elements and interlandmark angles. Animal Cognition, 2, 73-78.

Cheng, K. (1999b). Landmark-based spatial search in honeybees: II. Using gaps and blocks. Animal Cognition, 2, 79-90.

Cheng, K. (1999c). Spatial generalization in honeybees confirms Shepard's law. Behavioural Processes, 44, 309-316.

Cheng, K. (2000a). How honeybees find a place: Lessons from a simple mind. Animal Learning \& Behavior, 28, 1-15.

Cheng, K. (2000b). Shepard's law supported by honeybees in spatial generalization. Psychological Science, 11, 403-408.

Cheng, K., Collett, T. S., Pickhard, A., \& Wehner, R. (1987). The use of visual landmarks by honeybees: Bees weight landmarks according to their distance from the goal. Journal of Comparative Physiology A, 161, 469-475.

Cheng, K., Collett, T. S., \& Wehner, R. (1986). Honeybees learn the colour of landmarks. Journal of Comparative Physiology A, 159, 69-73.

Cheng, K., \& Spetch, M. L. (2000). Spatial peak shift and generalization in humans. Unpublished manuscript.

Cheng, K., Spetch, M. L., \& Johnston, M. (1997). Spatial peak shift and generalization in pigeons. Journal of Experimental Psychology: Animal Behavior Processes, 23, 469-481.

Cheng, K., Spetch, M. L., \& Miceli, P. (1996). Averaging temporal duration and spatial position. Journal of Experimental Psychology: Animal Behavior Processes, 22, 175-182.

Collett, T. S., \& BAron, J. (1994). Biological compasses and the coordinate frame of landmark memories in honeybees. Nature, $\mathbf{3 6 8}$, 137-140.

Collett, T. S., \& Rees, J. A. (1997). View-based navigation in hymenoptera: Multiple strategies of landmark guidance in the approach to a feeder. Journal of Comparative Physiology A, 181, 47-58.

Collett, T. S., \& ZeIL, J. (1998). Places and landmarks: An arthropod perspective. In S. Healy (Ed.), Spatial representation in animals (pp. 1853). Oxford: Oxford University Press.

Couvillon, P. A., Arakaki, L., \& Bitterman, M. E. (1997). Intramodal blocking in honeybees. Animal Learning \& Behavior, 25, 277-282.

Couvillon, P. A., \& Bitterman, M. E. (1989). Reciprocal overshadowing in the discrimination of color-odor compounds by honeybees: Further tests of a continuity model. Animal Learning \& Behavior, 17, 213-222.

Couvillon, P. A., \& Bitterman, M. E. (1999, November). Intermodal blocking in honeybees. Paper presented at the 40th Annual Meeting of the Psychonomic Society, Los Angeles.

Couvillon, P. A., Klosterhalfen, S., \& Bitterman, M. E. (1983). Analysis of overshadowing in honeybees. Journal of Comparative Psychology, 97, 154-166.

Couvillon, P. A., Mateo, E. T., \& Bitterman, M. E. (1996). Reward and learning in honeybees: Analysis of an overshadowing effect. Animal Learning \& Behavior, 24, 19-27.

Denniston, J. C., Miller, R. R., \& Matute, H. (1996). Biological sig- 
nificance as a determinant of cue competition. Psychological Science, 7, 325-331.

DYER, F. C. (1991). Bees acquire route-based memories but not cognitive maps in a familiar landscape. Animal Behaviour, 41, 239-246.

Frier, H. J., Edwards, E., Smith, C., Neal, S., \& Collett, T. S. (1996). Magnetic compass cues and visual pattern learning in honeybees. Journal of Experimental Biology, 199, 1353-1361.

Funayama, E. S., Couvillon, P. A., \& Bitterman, M. E. (1995). Compound conditioning in honeybees: Blocking tests of the independence assumption. Animal Learning \& Behavior, 23, 429-437.

Gallistel, C. R. (1990). The organization of learning. Cambridge MA: MIT Press.

Hammer, M., \& Menzel, R. (1995). Learning and memory in the honeybee. Journal of Neuroscience, 15, 1617-1630.

Hanson, H. M. (1959). Effects of discrimination training on stimulus generalization. Journal of Experimental Psychology, 58, 321-334.

HEALY, S. (ED.) (1998). Spatial representation in animals. Oxford: Oxford University Press.

Kamin, L. J. (1969). Selective association and conditioning. In N. J. Mackintosh \& W. K. Honig (Eds.), Fundamental issues in associative learning (pp. 42-64). Halifax, NS: Dalhousie University Press.

Kaye, H., Gambini, B., \& Mackintosh, N. J. (1988). A dissociation between one-trial overshadowing and the effect of a distractor on habituation. Quarterly Journal of Experimental Psychology, 40B, 31-47.

Leonard, B., \& McNaUghton, B. L. (1990). Spatial representation in the rat: Conceptual, behavioural, and neurophysiological perspectives. In R. P. Kesner \& D. S. Olton (Eds.), Neurobiology of comparative cognition (pp. 363-422). Hillsdale, NJ: Erlbaum.

MAcKInTOSH, N. J. (1976). Overshadowing and stimulus intensity. Animal Learning \& Behavior, 4, 186-192.

March, J., Chamizo, V. D., \& Mackintosh, N. J. (1992). Reciprocal overshadowing between intra-maze and extra-maze cues. Quarterly Journal of Experimental Psychology, 45B, 49-63.

Miller, R. R. \& Matute, H. (1996). Biological significance in forward and backward blocking: Resolution of a discrepancy between animal conditioning and human causal judgment. Journal of Experimental Psychology: General, 125, 370-386.

Morris, R. G. M. (1981). Spatial localization does not require the presence of local cues. Learning \& Motivation, 12, 239-260.

Morris, R. G. M., Garrud, P., Rawlins, J. N. P., \& O'Keefe, J. (1982). Place navigation in rats with hippocampal lesions. Nature, 297, 681-683.
Oberling, P., Bristol, A. S., Matute, H., \& Miller, R. R. (2000). Biological significance attenuates overshadowing, relative validity, and degraded contingency effects. Animal Learning \& Behavior, 28, 172-186.

O'KeEFe, J., \& NADEL, L. (1978). The hippocampus as a cognitive map. Oxford: Oxford University Press.

RILLING, M. (1977). Stimulus control and inhibitory processes. In W. K. Honig \& J. E. R. Staddon (Eds.), Handbook of operant behavior (pp. 432-480). Englewood Cliffs, NJ: Prentice-Hall.

Roberts, A. D. L., \& Pearce,J. M. (1999). Blocking in the Morris swimming pool. Journal of Experimental Psychology: Animal Behavior Processes, 25, 225-235.

Rodrigo, T., Chamizo, V. D., McLaren, I. P. L., \& Mackintosh, N. J. (1997). Blocking in the spatial domain. Journal of Experimental Psychology: Animal Behavior Processes, 23, 110-118.

Sánchez-Moreno, J., Rodrigo, T., Chamizo, V. D., \& Mackintosh, N. J. (1999). Overshadowing in the spatial domain. Animal Learning \& Behavior, 27, 391-398.

SHEPARD, R. N. (1958). Stimulus and response generalization: Deduction of the generalization gradient from a trace model. Psychological Review, 65, 242-256.

SHEPARD, R. N. (1987). Toward a universal law of generalization for psychological science. Science, 237, 1317-1323.

Sherry, D. F., \& VACCARINO, A. L. (1989). Hippocampus and memory for caches in black-capped chickadees. Behavioral Neuroscience, 103, 308-318.

Smith, B. H., \& Cobey, S. (1994). The olfactory memory of the honeybee Apis mellifera: II. Blocking between odorants in binary mixtures. Journal of Experimental Biology, 195, 91-108.

Spetch, M. L. (1995). Overshadowing in landmark learning: Touchscreen studies with pigeons and humans. Journal of Experimental Psychology: Animal Behavior Processes, 21, 166-181.

Wehner, R., Bleuler, S., Nievergelt, C., \& Shah, D. (1990). Bees navigate by using vectors and routes rather than maps. Naturwissenschaften, 77, 470-482.

Wehner, R, Lehrer, M., \& Harvey, P. (Eds.) (1996). Navigation. Journal of Experimental Biology, 199, 1-261.

Wehner, R, \& Menzel, R. (1990). Do insects have cognitive maps? Annual Review of Neuroscience, 13, 403-414.

(Manuscript received March 24, 1999; revision accepted for publication October 16, 2000.) 